\title{
Mechanical Property Analysis of Circular Polymer Membrane under Uniform Pressure
}

\author{
Sang Jianbing, Li Xiang, Xing Sufang, and Wang Wenjia \\ School of Mechanical Engineering, Hebei University of Technology, Tianjin 300130, China \\ Correspondence should be addressed to Sang Jianbing; sangjianbing@126.com
}

Received 15 May 2017; Revised 23 June 2017; Accepted 3 July 2017; Published 28 August 2017

Academic Editor: Angel Concheiro

Copyright (C) 2017 Sang Jianbing et al. This is an open access article distributed under the Creative Commons Attribution License, which permits unrestricted use, distribution, and reproduction in any medium, provided the original work is properly cited.

\begin{abstract}
Mechanical property analysis of circular hyperelastic polymer membrane under uniform pressure has been researched in this work. The polymer membrane material is assumed to be homogeneous and isotropic and incompressibility of materials has been considered. Based on the modified stain energy function from Gao and nonmomental theory of axial symmetry thin shell, finite deformation analysis of polymer membrane under uniform pressure has been proposed in current configuration and governing equations of polymer membrane have been achieved. By utilizing the boundary condition, theoretical results of governing equations have been obtained and vertical displacement distribution and stress distribution have been achieved. The results show that the constitutive parameter $n$ has a strengthening effect on the polymer material and the constitutive parameter $\alpha$ plays a controlling role for the second strain invariant $I_{2}$, which also has a strengthening effect on the polymer material. This research has revealed the deformational mechanism of polymer membrane and provided reference for the design of polymer membrane.
\end{abstract}

\section{Introduction}

Large deformation of membrane structures has been the subject of extensive research in recent years due to their applicability in numerous fields. Because they are of much lower weight and cost, they are ideally suited for applications in terrestrial and space structures [1]. Some other common applications include scientific ballooning, shock and vibration absorbers, thermal shields, and bioengineering and medical devices. Polymer membranes exhibit a number of interesting and counterintuitive phenomena on account of material and geometric nonlinearities. The analysis of polymer membranes under large deformation is usually based on the pioneering work of Green and Adkins [2] on nonlinear elasticity. Since then many theoretical and numerical works have been published, most of which deal with the large deformation and stability of polymer membranes under loads.

The general theory for large deformations polymer or rubber has been researched extensively in the past. Researches on the finite deformation of polymer membranes of various geometries are also available. Patil and DasGupta
[3] have researched on the inflation and interaction mechanics of a flat circular membrane inside an elastic cone under the action of uniform gas pressure. Tamadapu and DasGupta $[4,5]$ have studied the finite inflation of a hyperelastic toroidal membrane with an initially circular cross-section under internal pressure. Peridynamic strain energy density functions have been developed for a neo-Hookean type membrane under equibiaxial, planar, and uniaxial loading conditions and numerical results have concerned the deformation of a membrane with a defect in the form of a hole, a crack, and a rigid inclusion by Bang and Madenci [6]. Meunier et al. [7] have proposed the processing, characterisation, and modelling of anisotropic hyperelastic membranes and the mechanical response is characterised by performing tensile tests combined with kinematic field measurements by Digital Image Correlation. Macleod and Bartlett [8] have proposed an experimental and numerical investigation of instructions for hyperelastic membrane inflation based on the fluid structure coupling to have a research on the effects of changing the designs of the membrane structure to resist high pressure. 
Due to the ability of being subjected to large deformations, polymer membrane demonstrates nonlinear features inherently. Therefore, the selection of strain energy density function is a vital problem of theoretical research $[9,10]$. Many attempts have been made to develop a theoretical stress-strain relation. Mooney [11] proposed a phenomenological model with two parameters based on the assumption of a linear relation between the stress and strain during simple shear deformation as shown:

$$
W=C_{1}\left(I_{1}-3\right)+C_{2}\left(I_{2}-3\right),
$$

in which $C_{1}$ and $C_{2}$ are material constants and $\alpha=C_{2} / C_{1}$.

In 1948, Rivlin [12] put forward the strain energy function model to the isotropic hyperelastic materials:

$$
W=\sum_{i, j=0}^{\infty} C_{i j}\left(I_{1}-3\right)^{i}\left(I_{2}-3\right)^{j},
$$

in which $C_{i j}$ stands for material constant; $I_{1}$ and $I_{2}$ are, respectively, the first and second invariants of the left CauchyGreen deformation tensor.

Later, Treloar [13] published a model based on the statistical theory, the so-called neo-Hookean material model with only one material parameter. Mooney and neo-Hookean strain energy functions have played an important role in the development of the nonlinear hyperelastic theory and its applications.

$$
W\left(I_{1}\right)=\frac{1}{2} n k T\left(I_{1}-3\right) .
$$

In 1972, Ogden [14, 15] proposed a strain energy function expressed in terms of principal stretches, which is probably the best known example for the principal stretch-based constitutive formulations consistent with the Valanis-Landel hypothesis.

$$
W=\sum_{n=1}^{N} \frac{\mu_{n}}{\alpha_{n}}\left[\lambda_{1}^{\alpha_{n}}+\lambda_{2}^{\alpha_{n}}+\lambda_{3}^{\alpha_{n}}-3\right],
$$

where $\mu_{n}$ and $\alpha_{n}$ are the material constants and must be determined by a nonlinear fitting on experimental data. When $n=1, \alpha_{1}=2$, the strain energy function defined in (4) becomes the so-called neo-Hookean train energy function.

During 1996, Gent publishes a short note [16] where he proposed a new constitutive equation for the nonlinear elastic behavior of rubber like materials. Due to its formal simplicity, this model reached a great popularity in various scientific communities interested in large elastic deformations of solids.

$$
W=-\frac{\mu}{2} J_{m} \ln \left(1-\frac{I_{1}-3}{J_{m}}\right),
$$

where $\mu$ is the shear modulus and $J_{m}$ is the constant limiting value for $I_{1}-3$. Since $W$ depends on the only first invariant of $\mathbf{B}$, the Gent model belongs to the class of the generalized neo-Hookean materials.

Gao proposed the following strain energy function [17] in 1997:

$$
W=A\left(I_{1}^{n}+I_{-1}^{n}\right),
$$

where $A$ and $n$ are material parameters.
Based on Gao's constitutive model, a modified strain energy function [18] for the incompressible rubber like materials has been proposed as follows:

$$
W=A\left[\left(I_{1}^{n}-3^{n}\right)+\alpha\left(I_{2}^{n}-3^{n}\right)\right] .
$$

From the new constitutive model, we can see that when $n=1$ and $\alpha=0$, it transforms to neo-Hookean model; when $n=1$, it transforms to Mooney-Rivlin model.

In this work, mechanical property analysis of circular hyperelastic polymer membrane under uniform pressure has been researched. The polymer membrane material is assumed to be a homogeneous and isotropic and incompressibility of materials has been considered. Based on the modified stain energy function from Gao and nonmomental theory of axial symmetry thin shell, finite deformation analysis of polymer membrane under uniform pressure has been proposed in current configuration and governing equations of polymer membrane have been achieved. By utilizing the boundary condition, theoretical results of governing equations have been obtained and vertical displacement distribution and stress distribution have been achieved. Since the ultimate objective of the analysis is to design polymer membrane structure, experiments and numerical simulations are necessary. So experiments of polymer membrane under uniform pressure should be designed and implemented in the future to verify the correctness of analysis. What is more, we will program the user subroutine of proposed strain energy function and have numerical simulation of polymer membrane under uniform pressure.

\section{Kinematics of Deformation}

For circular polymer membrane is fixed around the circumference and sustained uniform normal pressure $q$, which is along the normal direction of polymer membrane as the deformation. Before deformation, the radius of circular polymer membrane is $a$ and thickness is $H$. After deformation, the circular membrane becomes rotational thin shell, and the thickness is $h$. Figures 1 and 2 give the geometrical relation of polymer membrane after deformation.

In the reference configuration, the geometry of the polymer membrane is described in terms of cylindrical polar coordinates $(R, \Theta, Z)$, and in the current coordinate, it is described in terms of cylindrical polar coordinates $(r, \theta, z)$; the principal stretch polymer membrane can be expressed as

$$
\begin{aligned}
& \lambda_{1}=\frac{d s}{d R}=\frac{d r}{\cos \varphi d R} ; \\
& \lambda_{2}=\frac{r}{R} ; \\
& \lambda_{3}=\frac{h}{H},
\end{aligned}
$$

in which $\lambda_{1}$ is the principal stretch along the tangential direction of longitude, $\lambda_{2}$ is the principal stretch along the tangential direction of latitude, and $\lambda_{3}(R)$ is the principal stretch along the normal direction of gyration shell, which is 


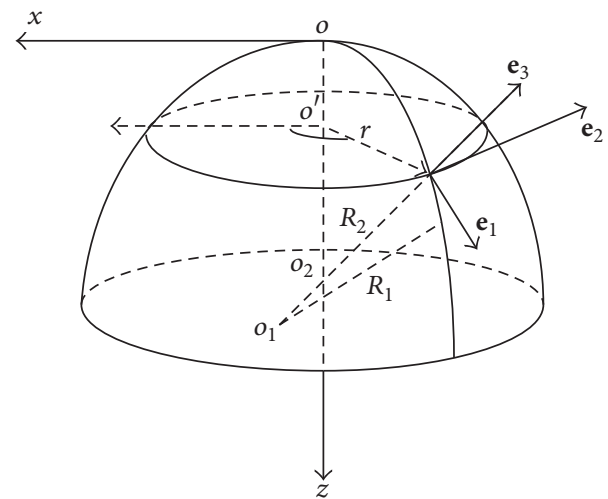

FIGURE 1: The diagram of deformed membrane.

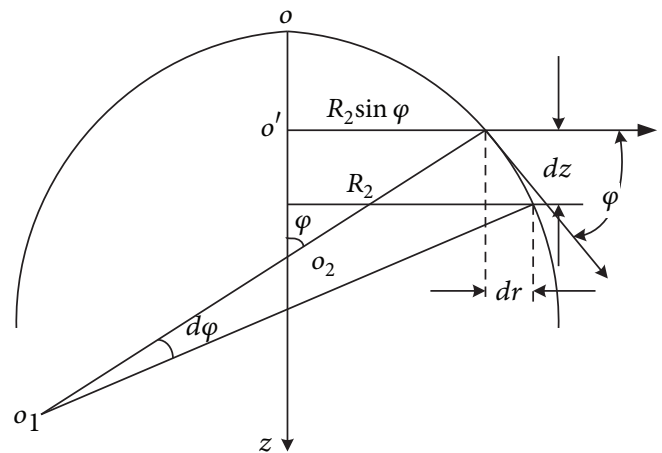

FIGURE 2: The geometric relation of rotational shell.

the function of $R$. If the thickness of polymer membrane is $H$ before deformation, then the thickness will be changed as

$$
h=h(R)=H \cdot \lambda_{3}(R) .
$$

Figure 3 shows the diagram of equilibrium relation. For thin shell, the equilibrium equation can expressed as

$$
\begin{aligned}
& \frac{1}{R_{1}} \frac{\partial N_{\varphi}}{\partial \varphi}+\frac{\cot \varphi}{R_{2}}\left(N_{\varphi}-N_{\theta}\right)+\frac{1}{R_{2} \sin \varphi} \frac{\partial N_{\varphi \theta}}{\partial \theta}+X \\
& =0 \\
& \frac{1}{R_{2} \sin \varphi} \frac{\partial N_{\theta}}{\partial \theta}+\frac{2 \cot \varphi}{R_{2}} N_{\varphi \theta}+\frac{1}{R_{1}} \frac{\partial N_{\varphi \theta}}{\partial \varphi}+Y=0, \\
& \frac{N_{\varphi}}{R_{1}}+\frac{N_{\theta}}{R_{2}}=Z=q .
\end{aligned}
$$

Considering the space axial asymmetry of polymer membrane after deformation, the equilibrium equation can be simplified as

$$
\begin{aligned}
\frac{1}{R_{1}} \frac{\partial N_{\varphi}}{\partial \varphi}+\frac{\cos \varphi}{R_{2} \sin \varphi}( & \left.N_{\varphi}-N_{\theta}\right)=0 \\
\frac{N_{\varphi}}{R_{1}}+\frac{N_{\theta}}{R_{2}} & =q .
\end{aligned}
$$

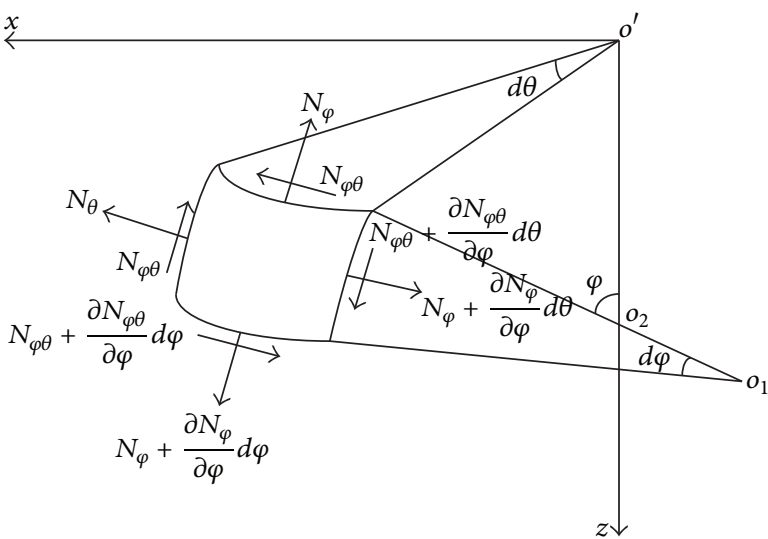

FIGURE 3: The diagram of equilibrium relation.

Based on Gauss-Kodak's relationship, the following expression can be achieved:

$$
\frac{d r}{d \varphi}=\frac{d\left(R_{2} \sin \varphi\right)}{d \varphi}=R_{1} \cos \varphi .
$$

Substituting (12) into (11), we can get

$$
\frac{d}{d r}\left(r N_{\varphi}\right)=N_{\theta} .
$$

Define $T_{1}$ and $T_{2}$, meet $T_{i}=H \lambda_{3} \sigma_{i}$, and substitute $N_{\varphi}$ and $N_{\theta}$ for $T_{1}$ and $T_{2}$; we can get

$$
\frac{d}{d r}\left(r T_{1}\right)=T_{2} .
$$

The first expression of (11) can be changed as

$$
T_{1} r \cos \varphi \frac{d \varphi}{d r}+T_{2} \sin \varphi=r q .
$$

\section{Constitutive Relation and \\ Governing Equation}

For isotropic materials, strain energy function can be represented in terms of invariants or principal stretches

$$
W=W\left(I_{1}, I_{2}, I_{3}\right),
$$

where $I_{1}, I_{2}$, and $I_{3}$ are the three strain invariants, which can be expressed as

$$
\begin{aligned}
& I_{1}=\lambda_{1}^{2}+\lambda_{2}^{2}+\lambda_{3}^{2}, \\
& I_{2}=\lambda_{1}^{2} \lambda_{2}^{2}+\lambda_{2}^{2} \lambda_{3}^{2}+\lambda_{3}^{2} \lambda_{1}^{2}, \\
& I_{3}=\lambda_{1}^{2} \lambda_{2}^{2} \lambda_{3}^{2} .
\end{aligned}
$$

Considering the conventional assumption of incompressibility for polymer membrane $J=\lambda_{1} \lambda_{2} \lambda_{3}=1$, we can get

$$
\begin{aligned}
& I_{1}=\lambda_{1}^{2}+\lambda_{2}^{2}+\frac{1}{\lambda_{1}^{2} \lambda_{2}^{2}}, \\
& I_{2}=\frac{1}{\lambda_{1}^{2}}+\frac{1}{\lambda_{2}^{2}}+\lambda_{1}^{2} \lambda_{2}^{2} .
\end{aligned}
$$


And the Cauchy stress from (7) can be expressed as

$$
\boldsymbol{\sigma}=-p \mathbf{I}+2 \frac{\partial W}{\partial I_{1}} \mathbf{B}-2 \frac{\partial W}{\partial I_{2}} \mathbf{B}^{-1}
$$

The left Cauchy-Green strain tensors $\mathbf{B}$ and $\mathbf{B}^{-1}$ have the following form:

$$
\begin{aligned}
\mathbf{B} & =\mathbf{F} \cdot \mathbf{F}^{T}=\lambda_{1}^{2} \mathbf{e}_{1} \otimes \mathbf{e}_{1}+\lambda_{2}^{2} \mathbf{e}_{2} \otimes \mathbf{e}_{2}+\lambda_{3}^{2} \mathbf{e}_{3} \otimes \mathbf{e}_{3}, \\
\mathbf{B}^{-1} & =\lambda_{1}^{-2} \mathbf{e}_{1} \otimes \mathbf{e}_{1}+\lambda_{2}^{-2} \mathbf{e}_{2} \otimes \mathbf{e}_{2}+\lambda_{3}^{-2} \mathbf{e}_{3} \otimes \mathbf{e}_{3} .
\end{aligned}
$$

Substitute (20) into (19); it can be achieved as

$$
\begin{aligned}
& \sigma_{1}=2 \lambda_{1}^{2} \frac{\partial W}{\partial I_{1}} W_{1}-2 \lambda_{1}^{-2} \frac{\partial W}{\partial I_{2}}-p, \\
& \sigma_{2}=2 \lambda_{2}^{2} \frac{\partial W}{\partial I_{1}}-2 \lambda_{2}^{-2} \frac{\partial W}{\partial I_{2}}-p, \\
& \sigma_{3}=2 \lambda_{3}^{2} \frac{\partial W}{\partial I_{1}}-2 \lambda_{3}^{-2} \frac{\partial W}{\partial I_{2}}-p .
\end{aligned}
$$

For thin polymer membrane, the radial and circumferential Cauchy stress is much larger than axial Cauchy stress; from (21) we can achieve

$$
p=2 \lambda_{3}^{2} W_{1}-2 \lambda_{3}^{-2} W_{2} .
$$

By utilizing $T_{i}=H \lambda_{3} \sigma_{i}$, the following expression can be achieved from (21):

$$
\begin{aligned}
& T_{1}=2 \lambda_{3} H\left(\lambda_{1}^{2}-\lambda_{3}^{2}\right)\left(W_{1}+\lambda_{2}^{2} W_{2}\right), \\
& T_{2}=2 \lambda_{3} H\left(\lambda_{2}^{2}-\lambda_{3}^{2}\right)\left(W_{1}+\lambda_{1}^{2} W_{2}\right) .
\end{aligned}
$$

in which $W_{1}=\partial W / \partial I_{1}$ and $W_{2}=\partial W / \partial I_{2}$

In common, large deformation analysis has been proposed in the reference coordinate system. Because the fundamental physical quantities are the functions of $R$ for spatial axisymmetric problem, from the geometrical relations, the following expression can be achieved:

$$
\cos \varphi=\frac{1}{\lambda_{1}} \frac{d r}{d R}
$$

The governing equations of polymer membrane can be obtained in reference configuration. However, the fundamental physical quantities can also be expressed based on the function of $r$ for a whole circular polymer membrane. That is, it is convenient for polymer membrane to be analyzed in the current coordinate system.

In current coordinate system, for a whole circle polymer membrane, substituting (14) into (15), we can get

$$
\begin{aligned}
\frac{d}{d r}\left(r T_{1} \sin \varphi\right) & =r q, \\
\sin \varphi & =\frac{r q}{2 T_{1}} .
\end{aligned}
$$

From the geometric relation of Figure 2, the following equations can be got:

$$
\begin{aligned}
\frac{d z}{d r} & =\tan \varphi, \\
\cos \varphi & =\frac{d r}{d s} .
\end{aligned}
$$

Based on the principal stretch polymer membrane of (8), we can get

$$
\frac{d \lambda_{2}}{d r}=\frac{\lambda_{2}}{r}\left(1-\frac{\lambda_{2}}{\lambda_{1} \cos \varphi}\right)
$$

Governing equations from Euler coordinate system can be achieved as

$$
\begin{aligned}
\frac{d}{d r}\left(r T_{1}\right) & =T_{2} ; \\
\frac{d z}{d r} & =\tan \varphi, \\
\frac{d \lambda_{2}}{d r} & =\frac{\lambda_{2}}{r}\left(1-\frac{\lambda_{2}}{\lambda_{1} \cos \varphi}\right) ; \\
\sin \varphi & =\frac{r q}{2 T_{1}} .
\end{aligned}
$$

In order to decrease the effect of dimension, we make $H=$ 1. Based on the first expression of (28) and (23), we can get

$$
\begin{aligned}
& \frac{d T_{1}}{d r}=\frac{1}{r}\left(T_{2}-T_{1}\right), \\
& T_{1}-T_{2}=\frac{2}{\lambda_{1} \lambda_{2}}\left[\left(\lambda_{1}^{2}-\lambda_{2}^{2}\right) W_{1}+\left(\frac{1}{\lambda_{2}^{2}}-\frac{1}{\lambda_{1}^{2}}\right) W_{2}\right],
\end{aligned}
$$

$T_{1}$

$$
=\frac{2}{\lambda_{1} \lambda_{2}}\left[\left(\lambda_{1}^{2}-\frac{1}{\lambda_{1}^{2} \lambda_{2}^{2}}\right) W_{1}+\left(\lambda_{1}^{2} \lambda_{2}^{2}-\frac{1}{\lambda_{1}^{2}}\right) W_{2}\right] .
$$

Define $\varphi_{1}=\left(\lambda_{1}^{4} \lambda_{2}^{2}-1\right) / \lambda_{1}^{3} \lambda_{2}^{3}$ and $\varphi_{2}=\left(\lambda_{1}^{4} \lambda_{2}^{2}-1\right) / \lambda_{1}^{3} \lambda_{2}$; the following expression can be got:

$$
\frac{d T_{1}}{d r}=\frac{d}{d r}\left(\varphi_{1} W_{1}+\varphi_{2} W_{2}\right) .
$$

From (30) and (18), we can get

$$
\begin{aligned}
& \frac{d \varphi_{1}}{d r}=A_{1} \frac{d \lambda_{1}}{d r}+A_{2} \frac{d \lambda_{2}}{d r} \\
& \frac{d \varphi_{2}}{d r}=A_{3} \frac{d \lambda_{1}}{d r}+A_{4} \frac{d \lambda_{2}}{d r} \\
& \frac{d I_{1}}{d r}=A_{5} \frac{d \lambda_{1}}{d r}+A_{6} \frac{d \lambda_{2}}{d r} \\
& \frac{d I_{2}}{d r}=A_{7} \frac{d \lambda_{1}}{d r}+A_{8} \frac{d \lambda_{2}}{d r}
\end{aligned}
$$


where

$$
\begin{aligned}
& A_{1}=\frac{\lambda_{1}^{4} \lambda_{2}^{2}+3}{\lambda_{1}^{4} \lambda_{2}^{3}}, \\
& A_{2}=\frac{3-\lambda_{1}^{4} \lambda_{2}^{2}}{\lambda_{1}^{3} \lambda_{2}^{4}}, \\
& A_{3}=\frac{\lambda_{1}^{4} \lambda_{2}^{2}+3}{\lambda_{1}^{4} \lambda_{2}}, \\
& A_{4}=\frac{\lambda_{1}^{4} \lambda_{2}^{2}+1}{\lambda_{1}^{3} \lambda_{2}^{2}}, \\
& A_{5}=2 \cdot \frac{\lambda_{1}^{4} \lambda_{2}^{2}-1}{\lambda_{1}^{3} \lambda_{2}^{2}}, \\
& A_{6}=2 \cdot \frac{\lambda_{1}^{2} \lambda_{2}^{4}-1}{\lambda_{1}^{2} \lambda_{2}^{3}}, \\
& A_{7}=2 \cdot \frac{\lambda_{1}^{4} \lambda_{2}^{2}-1}{\lambda_{1}^{3}}, \\
& A_{8}=2 \cdot \frac{\lambda_{1}^{2} \lambda_{2}^{4}-1}{\lambda_{2}^{3}} .
\end{aligned}
$$

Substituting (31) into (30), the following expression can be achieved:

$$
\begin{aligned}
\frac{d T_{1}}{d r} & =\frac{d}{d r}\left(\varphi_{1} W_{1}+\varphi_{2} W_{2}\right) \\
= & \left(W_{1} A_{1}+W_{2} A_{3}+\varphi_{1} W_{11} A_{5}+\varphi_{2} W_{22} A_{7}\right) \frac{d \lambda_{1}}{d r} \\
& +\left(W_{1} A_{2}+W_{2} A_{4}+\varphi_{1} W_{11} A_{6}+\varphi_{2} W_{22} A_{8}\right) \frac{d \lambda_{2}}{d r} .
\end{aligned}
$$

Define $\psi_{1}=W_{1} A_{1}+W_{2} A_{3}+\varphi_{1} W_{11} A_{5}+\varphi_{2} W_{22} A_{7}$ and $\psi_{2}=W_{1} A_{2}+W_{2} A_{4}+\varphi_{1} W_{11} A_{6}+\varphi_{2} W_{22} A_{8}$; the first expression of (29) can be expressed as

$$
\psi_{1} \frac{d \lambda_{1}}{d r}+\psi_{2} \frac{d \lambda_{2}}{d r}=-\frac{\psi}{r}
$$

The differential equations of polymer membrane under vertical pressure can be got as

$$
\begin{aligned}
\frac{d \lambda_{1}}{d r} & =\frac{\psi_{2} \lambda_{2} \lambda_{2}}{r \psi_{1} \lambda_{1} \cos \varphi}-\frac{\psi_{2} \lambda_{2}+\psi}{r \psi_{1}}, \\
\frac{d \lambda_{2}}{d r} & =\frac{\lambda_{2}}{r}\left(1-\frac{\lambda_{2}}{\lambda_{1} \cos \varphi}\right), \\
\frac{d z}{d r} & =\tan \varphi \\
\sin \varphi & =\frac{r q}{2 T_{1}}
\end{aligned}
$$

in which $W_{11}=\partial^{2} W / \partial I_{1}^{2}, W_{22}=\partial^{2} W / \partial I_{2}^{2}$. Based on the modified strain energy function (7), we can get $W_{12}=W_{21}=$ $\partial^{2} W / \partial I_{1} \partial I_{2}=0$.
TABLE 1: The initial value of governing equations with the variation of $q / A$.

\begin{tabular}{cccc}
\hline$n$ & $\alpha$ & $\frac{q}{A}$ & $\begin{array}{c}\lambda_{1}=\lambda_{2}=x_{0} \\
\text { initial value at } r=0\end{array}$ \\
\hline 2 & 1 & 7.5 & 1.0765 \\
2 & 1 & 10 & 1.0954 \\
2 & 1 & 15 & 1.1300 \\
\hline
\end{tabular}

TABLE 2: The initial value of governing equations with the variation of $n$.

\begin{tabular}{lccc}
\hline$n$ & $\alpha$ & $\frac{q}{A}$ & $\begin{array}{c}\lambda_{1}=\lambda_{2}=x_{0} \\
\text { initial value at } r=0\end{array}$ \\
\hline 1.5 & 0.6 & 10 & 1.0619 \\
2 & 0.6 & 10 & 1.1118 \\
2.5 & 0.6 & 10 & 1.2228 \\
\hline
\end{tabular}

TABLE 3: The initial value of governing equations with the variation of $\alpha$.

\begin{tabular}{cccc}
\hline$n$ & $\alpha$ & $\frac{q}{A}$ & $\begin{array}{c}\lambda_{1}=\lambda_{2}=x_{0} \\
\text { initial value at } r=0\end{array}$ \\
\hline 2 & 0 & 10 & 1.1382 \\
2 & 0.3 & 10 & 1.1277 \\
2 & 0.6 & 10 & 1.1118 \\
2 & 1 & 10 & 1.0954 \\
\hline
\end{tabular}

\section{Theoretical Results and Discussions}

For circular polymer membrane, it is fixed around the circumference and sustained uniform spatial axisymmetric pressure, from which we can get $\varphi=0, z=0$ when $r=0$. By utilizing this relation, the following expression can be achieved:

$$
\lambda_{1}=\lambda_{2}
$$

At the fixed boundary of polymer membrane, we can get $\lambda_{2}=1$ when $r=a$. In order to analyze conveniently, no-dimension analysis has been proposed. $R / a$ and $r / a$ are adopted to express the radical position of polymer membrane before deformation and after deformation.

In order to make the no-dimension analysis of $z, q, T_{1}$, and $T_{2}$, the expressions of $z / a, q / A, T_{1} / A$, and $T_{2} / A$ have been adopted in the analysis. For given material parameters and external load of $n, \alpha$, and $q / A$, initial value $\lambda_{1}=\lambda_{2}=x_{0}$ has been determined based on the method of shooting method, at the central point of $r=0$. Then by utilizing the Runge-Kutta method, the relation curves of $\sigma_{1} / A, \sigma_{2} / A$, and $z / a$ have been achieved.

Tables 1-3 show the initial value of governing equations with the variation of $q / A, n$, and $\alpha$.

By utilizing the initial values, the governing equation (27) has been solved to find out the precise value to meet $\lambda_{2}=1$ at the fixed boundary $r / a=1$. Thus, the numerical results meet all boundary conditions. Figures 4-6 show the vertical displacement curve, the radical stress distribution curve, and the circumferential stress distribution 


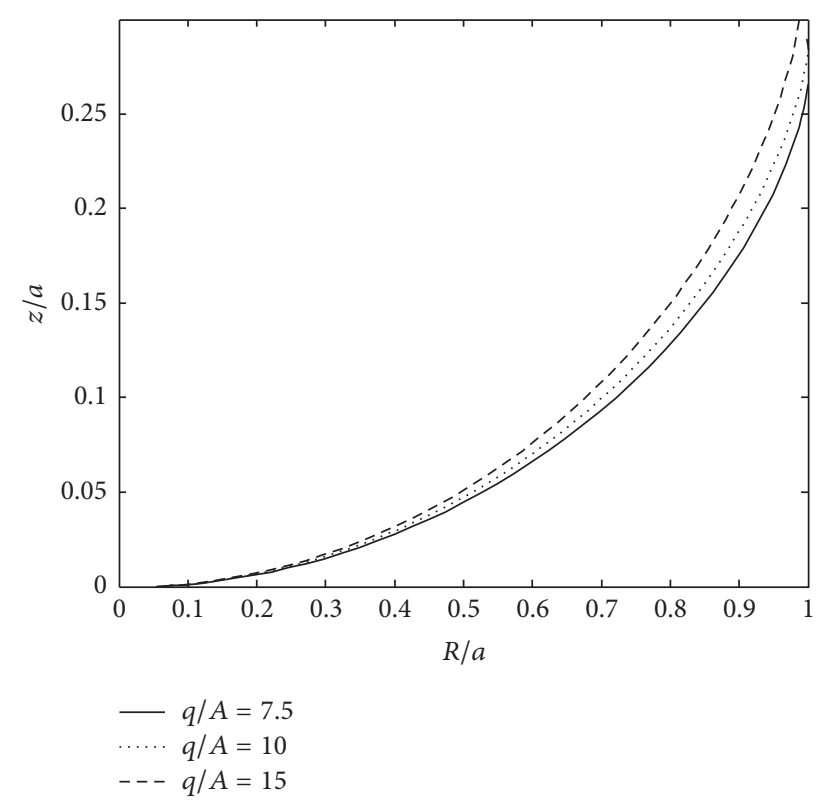

Figure 4: The vertical displacement curve of membrane $(n=2 ; \alpha=$ $1)$.

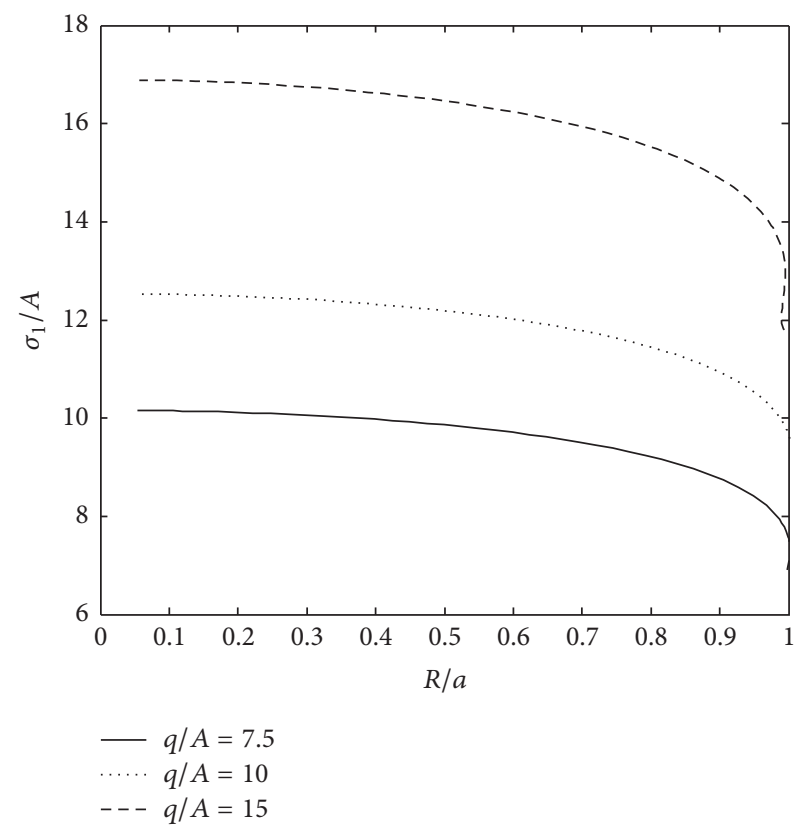

FIGURE 5: The radical stress distribution curve on membrane $(n=2$; $\alpha=1)$.

curve correspondingly with different vertical pressure, with fixed material parameters $n=2$ and $\alpha=1$. From Figure 4, we can see that vertical deformation increases gradually in the direction of the radius of polymer membrane with the increase of vertical pressure. But from Figures 5 and 6, it can be seen that the radical stress and the circumferential stress decrease gradually in the direction of the radius of polymer membrane and increase gradually as the vertical pressure increases with the increase of vertical pressure, which is

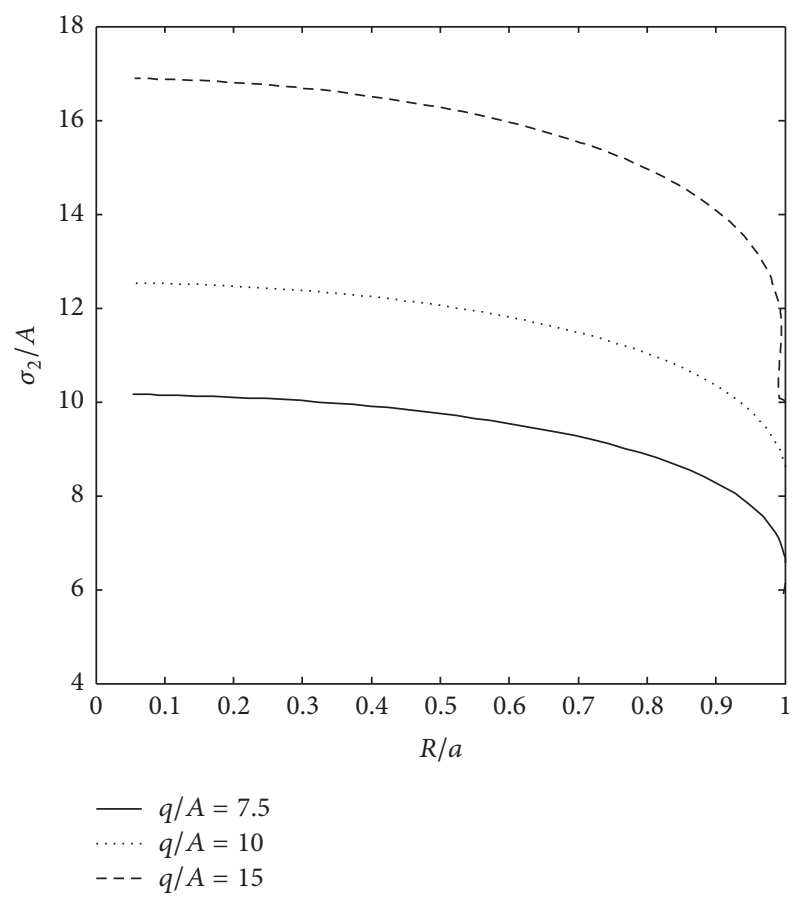

FIgURE 6: The circumferential stress distribution curve on membrane $(n=2 ; \alpha=1)$.

according to the practical deformation. At the point $z=0$, the radical stress and circumferential stress reach the maximal value.

When constitutive parameter $\alpha=0.6$ and external load $q / A=10$, analysis of polymer membrane has been proposed based on different constitutive parameter $n$. Figures 7,9 , and 11 show the vertical displacement curve, the radical stress distribution curve, and the circumferential stress distribution curve correspondingly with different constitutive parameter $n$ with fixed $q / A=10$ and $\alpha=0.6$. As can be noted in Figure 7, vertical deformation increases gradually in the direction of the radius of polymer membrane with the increase of vertical pressure; however, it decreases as the constitutive parameter $n$ increase. This means the deformation of polymer membrane is apparently affected by the constitutive parameter $n$. Figures 9 and 11 display the variation of radical stress and circumferential stress along the direction of the radius of polymer membrane, from which we can see that the radical and circumferential stress decrease gradually in the direction of the radius of polymer membrane and the constitutive parameter $n$ has a significant effect on the radical stress and circumferential stress. At the point $z=0$, the radical stress is equal to the circumferential stress and the value reaches the maximal value. The radical and circumferential stress reach the minimal value at the fixed boundary $r=a$ and their value is not equal to zero. This indicates that the stiffness of polymer membrane can be enhanced gradually as the increase of constitutive $n$, which improves the deformational ability of polymer membrane and has a major impact on the mechanical property of polymer membrane. 


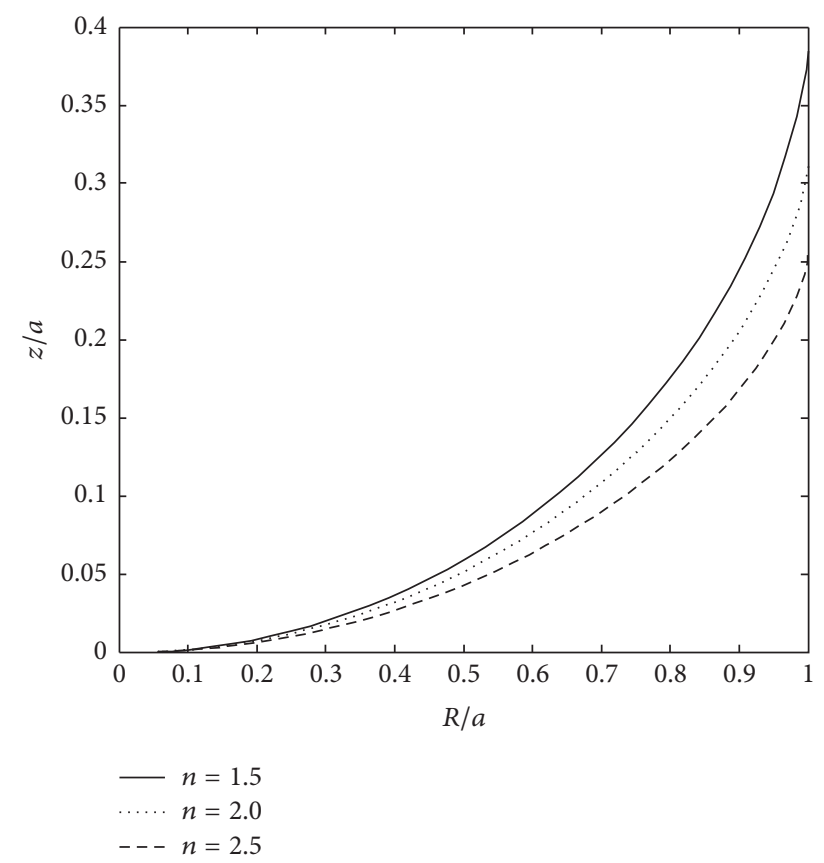

Figure 7: The vertical displacement curve of polymer membrane $(q / A=10 ; \alpha=0.6)$.

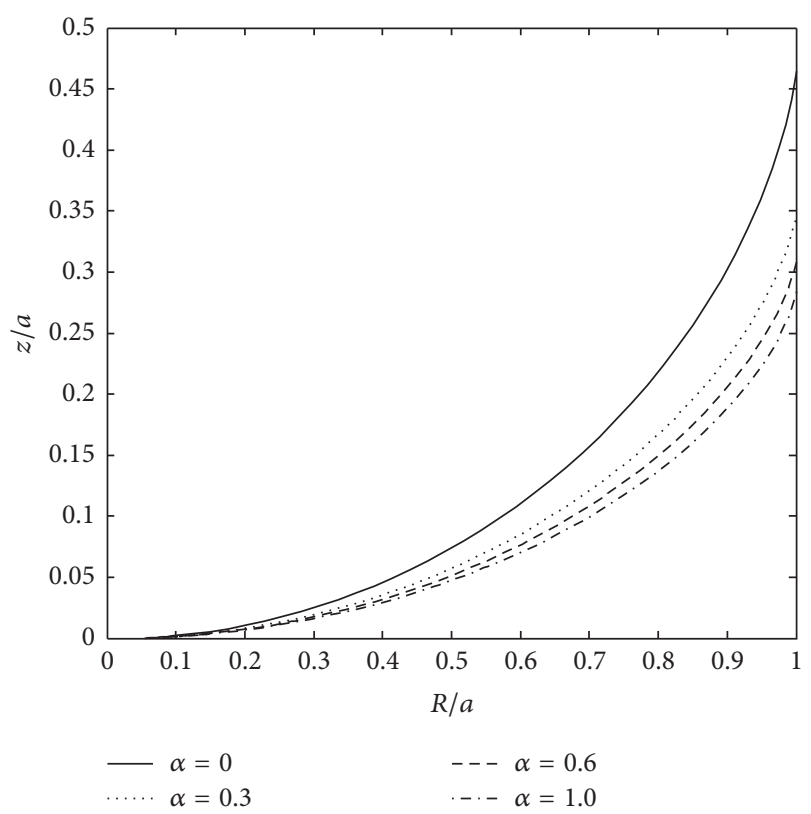

Figure 8: The vertical displacement curve of polymer membrane $(q / A=10 ; n=2)$.

In the meanwhile, when constitutive parameter $n=2$ and external load $q / A=10$, analysis of polymer membrane has been proposed based on different constitutive parameter $\alpha$. Figures 8,10 , and 12 show the vertical displacement curve, the radical stress distribution curve, and the circumferential stress distribution curve correspondingly with different constitutive parameter $n$ with fixed $q / A=10$ and $\alpha=0.6$.

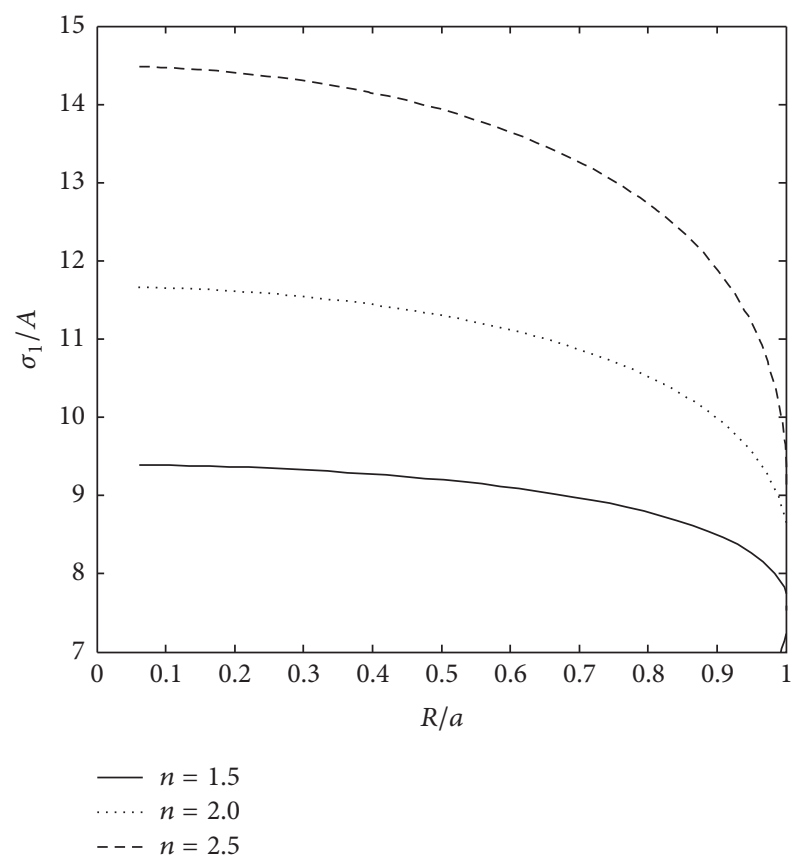

FIGURE 9: The radical stress distribution on membrane $(q / A=10$; $\alpha=0.6)$.

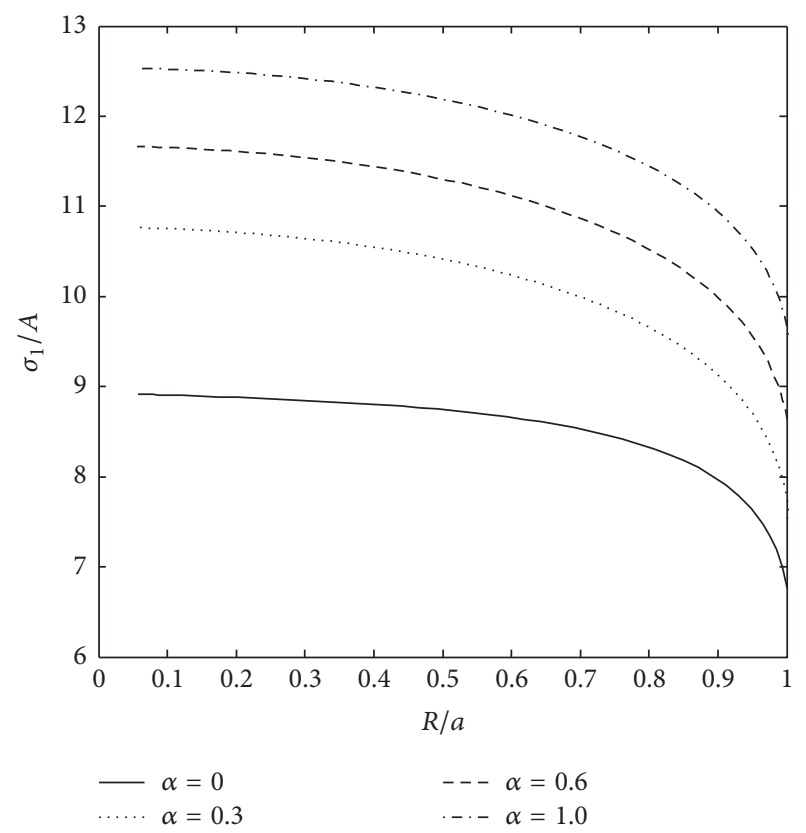

FIgURE 10: The radical stress distribution on membrane $(q / A=10$; $n=2)$.

As shown in Figure 8, vertical deformation increases gradually in the direction of the radius of polymer membrane with the increase of vertical pressure; however, it decreases as the constitutive parameter $\alpha$ increases. Figures 10 and 12 display the variation of radical stress and circumferential stress along the direction of the radius of polymer membrane, from which we can see that the radical and circumferential 


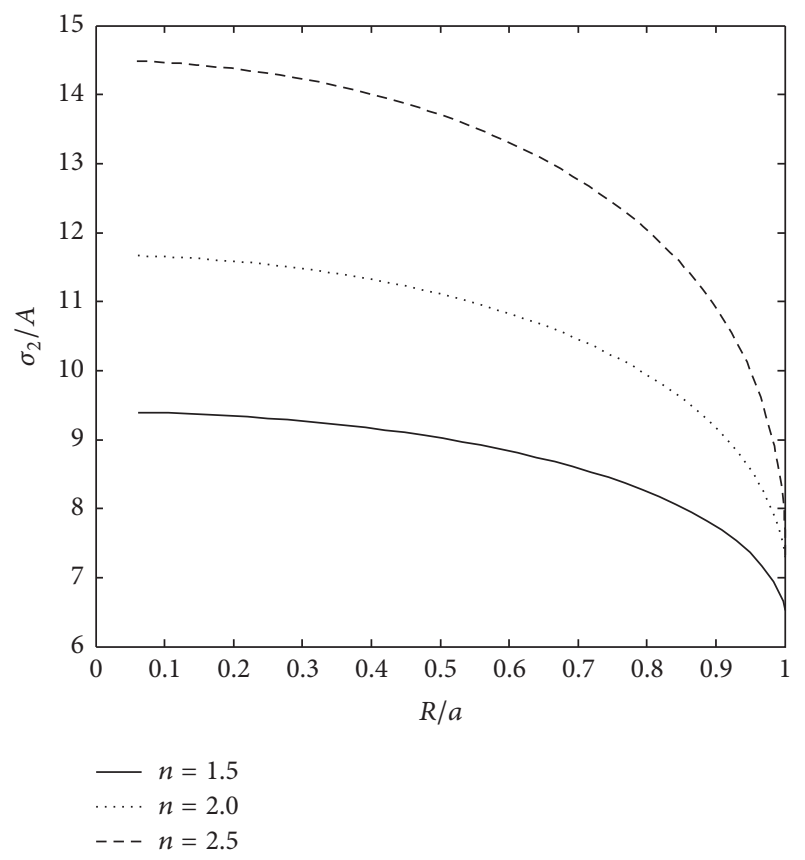

FIGURE 11: The circumferential stress distribution on membrane $(q / A=10 ; \alpha=0.6)$.

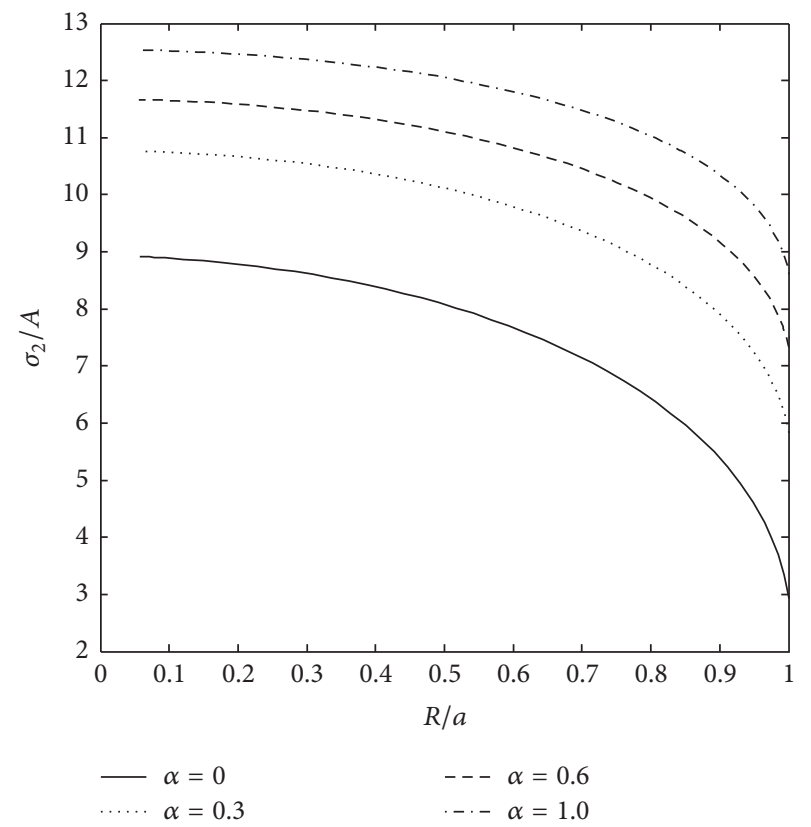

FIgURE 12: The circumferential stress distribution on membrane $(q / A=10 ; n=2)$.

stress decrease gradually in the direction of the radius of polymer membrane and the constitutive parameter $\alpha$ also has a significant effect on the radical stress and circumferential stress. This indicates that the stiffness of polymer membrane can be enhanced gradually as the increase of constitutive $\alpha$, which improves the deformational ability of polymer membrane and also has a major impact on the mechanical property of polymer membrane.

\section{Conclusion}

A modified strain energy function from Gao has been utilized to have a research on the large deformation problem of circular polymer membrane under uniform pressure. Based on the stain energy function from Gao and nonmomental theory of axial symmetry thin revolving shell, the theoretical results of governing equations have been obtained and vertical displacement distribution and stress distribution have been achieved. The results show that the vertical deformation increases gradually in the direction of the radius of polymer membrane with the increase of vertical pressure; however, it decreases as the constitutive parameters $n$ and $\alpha$ increase. The radical and circumferential stress decrease gradually in the direction of the radius of polymer membrane. The constitutive parameters $n$ and $\alpha$ both have a significant effect on the radical stress and circumferential stress. It means that the constitutive parameter $n$ has a strengthening effect on the polymer material and the constitutive parameter $\alpha$ plays a controlling role for the second strain invariant $I_{2}$, which also has a strengthening effect on the polymer material.

\section{Conflicts of Interest}

The authors declare that there are no conflicts of interest regarding the publication of this paper.

\section{Acknowledgments}

This paper is supported by Project of Tianjin Excellent Science and Technology Correspondent (Grant no. 16JCTPJC53100), Scientific Research Key Project of Hebei Province Education Department (Grand no. ZD2016083), and Natural Science Foundation of Hebei Province (Grant nos. A2015202242 and A2016202195).

\section{References}

[1] H. Sakamoto, M. C. Natori, S. Kadonishi et al., "Folding patterns of planar gossamer space structures consisting of membranes and booms," Acta Astronautica, vol. 94, pp. 34-41, 2014.

[2] A. E. Green and J. E. Adkins, Large Elastic Deformations, and Non-Linear Continuum Mechanics, Clarendon Press, Oxfordm, UK, 1960.

[3] A. Patil and A. DasGupta, "Constrained inflation of a stretched hyperelastic membrane inside an elastic cone," Meccanica. International Journal of Theoretical and Applied Mechanics, vol. 50, no. 6, pp. 1495-1508, 2015.

[4] G. Tamadapu and A. DasGupta, "Finite inflation analysis of a hyperelastic toroidal membrane of initially circular crosssection," International Journal of Non-Linear Mechanics, vol. 49, pp. 31-39, 2013.

[5] G. Tamadapu and A. Dasgupta, "Finite inflation of a hyperelastic toroidal membrane over a cylindrical rim," International Journal of Solids and Structures, vol. 51, no. 2, pp. 430-439, 2014.

[6] D. J. Bang and E. Madenci, "Peridynamic modeling of hyperelastic membrane deformation," Journal of Engineering Materials and Technology, Transactions of the ASME, vol. 139, no. 3, Article ID 031007, 2017. 
[7] L. Meunier, G. Chagnon, D. Favier et al., "Anisotropic large deformation of geometrically architectured unfilled silicone membranes," European Journal of Mechanics. A. Solids, vol. 50, pp. 87-99, 2015.

[8] S. Macleod and N. R. Bartlett, "Experimental and numerical investigation of instructions for hyperelastic membrane inflation using fluid structure coupling," Computer Modeling in Engineering \& Sciences, vol. 77, no. 3, pp. 183-200, 2011.

[9] U. K. Chakravarty and R. Albertani, "Experimental and finite element modal analysis of a pliant elastic membrane for micro air vehicles applications," Journal of Applied Mechanics, Transactions ASME, vol. 79, no. 2, Article ID 021004, 2012.

[10] H. Zahedmanesh, D. John Kelly, and C. Lally, "Simulation of a balloon expandable stentin a realistic coronary arterydetermination of the optimum modellingstrategy," Journal of Biomechanics, vol. 43, no. 11, pp. 2126-2132, 2010.

[11] M. Mooney, "A theory of large elastic deformation," Journal of Applied Physics, vol. 11, no. 9, pp. 582-592, 1940.

[12] R. S. Rivlin, "Large elastic deformations of isotropic materials. I. fundamental concepts," Philosophical Transactions of the Royal Society of London. Series A. Mathematical and Physical Sciences, vol. 240, pp. 459-490, 1948.

[13] L. R. G. Treloar, "The elasticity of a network of long-chain molecules," Transactions of the Faraday Society, vol. 42, pp. 8394, 1946.

[14] R. W. Ogden, "Large deformation isotropic elasticity-on the correlation of theory and experiment for the incompressible rubber-like solids," Proceedings of the Royal Society of London A: Mathematical A, vol. 326, no. 1567, pp. 565-584, 1972.

[15] R. W. Ogden, "Large deformation isotropic elasticity-on the correlation of theory and experiment for the compressible rubber-like solids," Proceedings of the Royal Society A: Mathematical, Physical and Engineering Sciences, vol. 328, no. 1575, pp. 567-583, 1972.

[16] A. N. A. Gent, "A new constitutive ralation for rubber," Rubber Chemistry Technology, vol. 69, no. 1, pp. 59-61, 1996.

[17] Y. C. Gao, "Large deformation field near a crack tip in rubberlike materials," Theoretical and Applied Fracture Mechanics, vol. 26, no. 3, pp. 155-162, 1997.

[18] J. B. Sang, L. F. Sun, and S. F. Xing, "Mechanical properties of polymer rubber materials based on a new constitutive model," Polymer \& Polymer Composite, vol. 22, no. 8, pp. 693-698, 2014. 

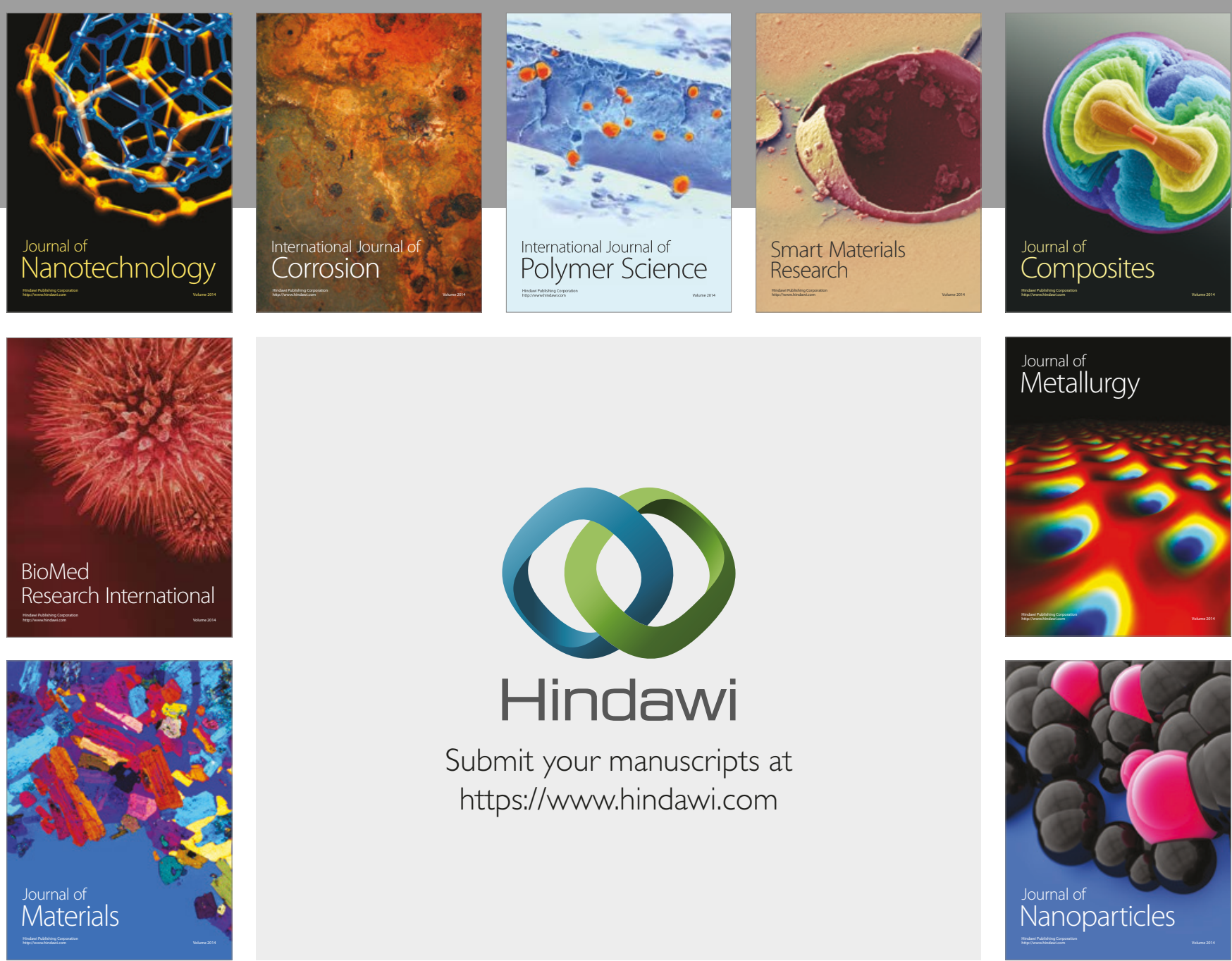

\section{Hindawi}

Submit your manuscripts at

https://www.hindawi.com
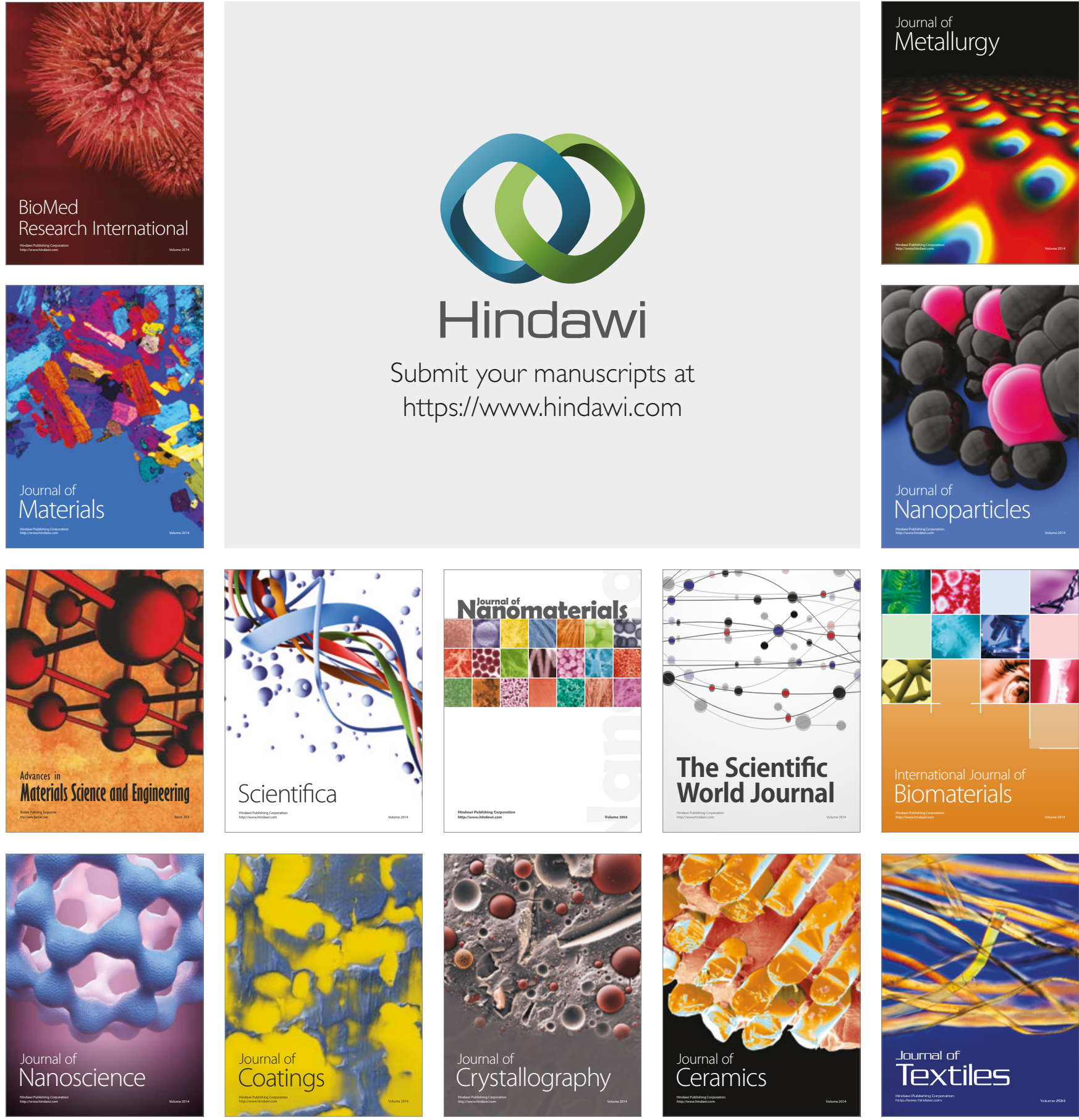

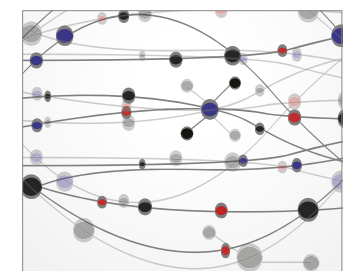

The Scientific World Journal
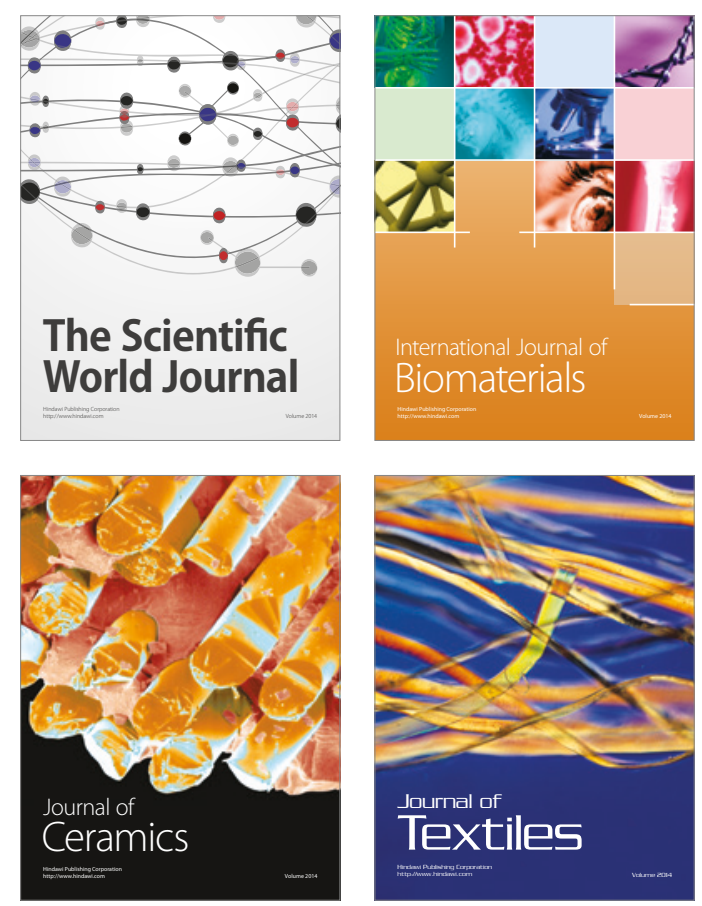